\section{End of Day Harvest Extends Shelf Life}

\author{
Graham J.J. Clarkson
}

School of Biological Sciences, University of Southampton, Bassett Crescent East, Southampton, SO16 7PX, UK

\author{
Steve D. Rothwell \\ Vitacress Salads Ltd., Lower Link Farm, St. Mary Bourne, Andover, SP11 \\ $6 D B, U K$
}

\author{
Gail Taylor ${ }^{1}$ \\ School of Biological Sciences, University of Southampton, Bassett Crescent \\ East, Southampton, SO16 7PX, UK
}

Additional index words. Lactuca sativa, Eruca vesicaria, cell wall extensibility, processability, xyloglucan endotransglucosylase

\begin{abstract}
Baby salad leaves of salad roquette, arugula in the U.S. (Eruca vesicaria ssp. sativa) had an increased postharvest shelf life of 2 to 6 days, while lollo rosso lettuce (Lactuca sativa L. 'Ravita') and red chard [Beta vulgaris L. var. flavescens (Lam.) Lam] baby salad leaves had increased shelf life of 1 to 2 days when harvested at the end of the day compared with leaves harvested at the start of the day. We have shown that improved shelf life of salad roquette and lollo rosso following end-of-day harvest was correlated with altered biophysical characteristics of the cell walls, with increased cell wall extensibility (percent plasticity and elasticity) measured at end of day. Leaf turgor pressure (P, MPa) was also highest in salad roquette and red chard at the end of day. Improved shelf life following 'end of day' harvest was also associated with the accumulation of leaf sucrose in salad roquette but not lollo rosso and red chard following daily photosynthesis. Diurnal alterations of leaf starch concentration were detected in lollo rosso and red chard but not in salad roquette. The degree of leaf shelf life extension in salad roquette and red chard was further associated with the peak rates of leaf photosynthetic activity. These data suggest that, depending on species, significant improvements to postharvest shelf life could be achieved through the rescheduling of time of day for harvest and also provide relevant information on the selection of traits for future genetic improvement.
\end{abstract}

The production of baby leaf salad crops for the prepacked market is now a global business. Baby salad leaves are harvested at an immature stage, when the leaves are $8( \pm 2)$ $\mathrm{cm}$ long, in comparison to the traditional head lettuce, and sold washed and ready to eat in a polyethylene bag. Specific breeding for baby salad varieties is on-going and along with qualities of taste and disease resistance it is of primary importance that all the leaf material for processing and packaging is of a suitably high quality. Therefore able to withstand the physical stresses involved in the process of washing, de-watering and packing without being injured, and thus be able to maintain a lengthy shelf life post processing without development of water-logging, discoloration or decay due to injuries sustained during processing. This quality we have termed the processability of the leaf (Clarkson et al., 2003).

In our previous work (Clarkson et al., 2003) we have examined material of varying processing quality to identify factors such as epidermal cell area and leaf biophysical

\footnotetext{
Received for publication 1 Nov. 2004. Accepted for publication 23 Jan. 2005. This work was supported by a scholarship to GJJC from Vitacress Salads Ltd. and the University of Southampton. The authors also acknowledge the contribution of members of G Taylor's research group in performing the UK harvest and the staff of Vitacress Kenya for their invaluable assistance.

${ }^{1}$ To whom reprint requests should be addressed; e-mail g.taylor@soton.ac.uk.
}

handling. A further potentially important factor is the time of day that the leaf is harvested. Current farming practice is to harvest leaves at first light allowing the crop to be shipped, processed or packed on the same day. However plant and leaf functional attributes are known to alter diurnally and this could impact on the processability traits of the leaves, although limited information is available to substantiate this point.

Considerable improvement in basil leaf shelf life when harvested at the end of the day versus in the early morning has been reported previously by Lange and Cameron (1994). Glasshouse grown sweet basil was harvested at set points during a 24-h period and the shelf life of the bagged product observed. Visual shelf life of sweet basil was shown to be increased by up to $170 \%$ when harvested at the end of the day (18:00 HR) in comparison to leaves harvested in the morning (06:00 HR). These dramatic changes in shelf life were attributed to diurnal changes in leaf status, but the causes of the changes were not determined, although the concentration of carbohydrate was proposed to have a role. Carbohydrates have also been implicated in chilling tolerance of tomato seedlings where King et al. (1988) stated that light acts to restore carbohydrates that are depleted overnight, improving seedling chilling tolerance.

The postharvest quality of lettuce cultivars has been shown to be affected by the time of harvest (Moccia et al., 1998) but the best time of day to harvest appeared variable. There was an influence of type on the optimum time to harvest, with the quality of a butterhead lettuce highest when harvested at 18:00 HR, but the Latin type lettuce exhibited best quality when harvested at 06:00 HR. This suggests an interaction between genotype and environment in determining postharvest quality, an area where further study is warranted. Noon was seen to be the worst time to harvest in both lettuce types. Carbohydrate concentration in field grown crisphead lettuces in California were shown to change in relation to an AM (07:00 HR) or PM (14:00 HR) harvest (Forney and Austin, 1988). Sucrose was higher at PM than AM, yet starch concentrations remained low and the monosaccharides glucose and fructose were highest when harvested at 07:00 HR, the point when carbon dioxide sensitivity was greatest.

The aim of the research presented here was to 1) discover the optimum time of harvest to improve the shelf life of baby leaf salad, across a range of baby leaf salad species, 2) determine the impact of the time of harvest on key traits of processability, 3) determine the mechanism underlying changes in shelf life, for contrasting leafy salad species using both field and controlled environment approaches. Our focus was on leaf photosynthesis and carbohydrate status, with measurements also on water relations and cell wall extensibility, since we have already shown that these are central in determining leaf processability (Clarkson et al., 2003).

\section{Materials and Methods}

Plantmaterial. Baby salad leaves produced commercially for use in the production of prepackaged salad mixes (leaf size 5 to $10 \mathrm{~cm}$ in length) were used in this study. Leaves tested were from lettuce, Lactuca sativa L. 'Ravita' (a loose leaf type lollo rosso) red chard, Beta vulgaris L. var. flavescens (Lam.) Lam and salad roquette or arugula, Eruca vesicaria ssp. sativa.

Field sites and controlled growth room environments. Two separate field sites and a controlled environment experiment were undertaken in the study. Experiment 1 was performed at Pinglestone Farm, near Winchester, U.K. (latitude $51^{\circ} 6$ ' N, longitude $1^{\circ} 10^{\prime} \mathrm{W}$ ) between 4 and 5 July 2001. Experiment 2 was at Lolomarik Farm, Timau, Kenya (latitude $0^{\circ} 5^{\prime} \mathrm{N}$, longitude $37^{\circ} 14^{\prime} \mathrm{E}$ ) between 2 and 23 Jan. 2002. It is in the rain shadow of Mount Kenya, at an approximate altitude of 2,500 m. Sampling in Expt. 1 was at the start of the U.K. summer, with only a short night 
of about $5 \mathrm{~h}$, the sampling period was dry and followed $24 \mathrm{~h}$ of clear, sunny weather (day and night temperature during the experiment about 23 and $10{ }^{\circ} \mathrm{C}$ respectively). Sampling in experiment 2 was during the dry season when Lolomarik Farm has a steady climate with $12 \mathrm{~h}$ of light and dark, warm days (about $25^{\circ} \mathrm{C}$ ), cool nights (about $6{ }^{\circ} \mathrm{C}$ ) and almost no rain.

Experiment 3 used two independent computer controlled growth rooms, with dark and light periods alternating between the rooms and conditions set to $27^{\circ} \mathrm{C}$ day, $13{ }^{\circ} \mathrm{C}$ night, with white fluorescent lighting at $170 \mu \mathrm{mol} \cdot \mathrm{m}^{-2} \cdot \mathrm{s}^{-1}$ to give12-h days. Seeds were germinated in 125$\mathrm{mm}^{3}$ cells using a compost mixture (medium grade 2 Vermiperl vermiculite : 1 Levingtons F2, v/v) (Avon Crop, Bracknell, U.K.). The trays were watered from below on a daily basis and the leaves were harvested once, 4 weeks post germination.

Experiment 1: U.K. field trial. Leaves of lollo rosso and salad roquette were cut in the field at 22:00 HR on 4 July 2001 and 10:00 HR on 5 July 2001 into polythene bags, within plastic crates and stored off the floor at $4{ }^{\circ} \mathrm{C}$ in a refrigerated unit. The baby salad was processed as described by Clarkson et al. (2003) on 6 July 2001 at 14:00 HR using a twin-tub washing machine that has been shown (data not presented) to replicate the degree of process injury caused by commercial wash lines. The product was weighed into 50g replications, kept in zip sealed, 180-gauge barrier polythene bags (Scientific Laboratory Supplies, Nottingham, U.K.) and stored at three temperatures $\left(3.5,6\right.$, and $\left.11 \pm 1{ }^{\circ} \mathrm{C}\right)$ with five replications at each temperature. Shelf life was determined by an independent panelist's daily examination of the bags at 14:00 HR, with rejection after two independent, distinguishable, signs of physiological or microbial breakdown in a bag such as water-logging, discoloration, decay and liquid leakage. At each harvest point, 50 leaves of each crop were sampled for biophysical testing, as Clarkson et al. (2003). Measurements of irreversible extensibility (percent plasticity) and reversible extensibility (percent elasticity) were extrapolated from chart recorder output.

Experiment 2: Kenyanfield trial. Baby leaves of red chard, lollo rosso and salad roquette were harvested in the same way as experiment 1 and stored at $4 \pm 0.5{ }^{\circ} \mathrm{C}$ ) in a refrigeration unit. Leaves were sampled at 18:30 HR on 8 Jan. 2002 and 06:15 HR on 9 Jan. 2002 (sundown and sunrise respectively) and processed at 15:00 HR on 9 Jan. 2002 along a staffed commercial packing line (Vitacress Kenya Ltd, Nairobi, Kenya). In total, $50 \mathrm{~g}$ of each crop was bagged in preformed commercial barrier salad film (35 $\mu \mathrm{m}$ orientated polypropylene with an antimist agent on the inside; Amcor Flexibles Europe Ledbury, Ledbury, U.K.) and heat sealed. Ten replications for each line were stored at $4 \pm 1$ ${ }^{\circ} \mathrm{C}$. Rejection was after only the first sign of breakdown in the bag following independent daily visual examination at 15:00 $\mathrm{HR}$.

Transpiration (E) and net photosynthetic assimilation rate (A) were recorded in growing baby salad leaves in the field between dawn and dusk on 14 Jan. 2002 for red chard and salad roquette. 10 unshaded outer leaves orientated to the sun were selected at each of six (06:45, 09:00, 12:00, 14:00, 16:00, and 18:30 HR) time points between sunrise and sundown and the data collected using a portable photosynthesis system(LI-6400; LI-COR, inc., Lincoln, Neb.). The infrared gas analysis two-chamber open system was used following the manufacturer's instructions with all settings to match the prevailing ambient conditions.

Leaves of red chard and salad roquette were selected with petioles to fit a 6-mm rubber gasket in the pressure chamber device and harvested at 18:30 HR on 16 Jan. 2002 and 06:30 HR on 17 Jan. 2002 into a humidified air-tight plastic box. Leaves were tested immediately for their water potential, $\Psi_{\mathrm{w}}$, using the pressure-bomb technique (Scholander et al., 1965) and leaf sap osmolarity as described in Clarkson et al. (2003)
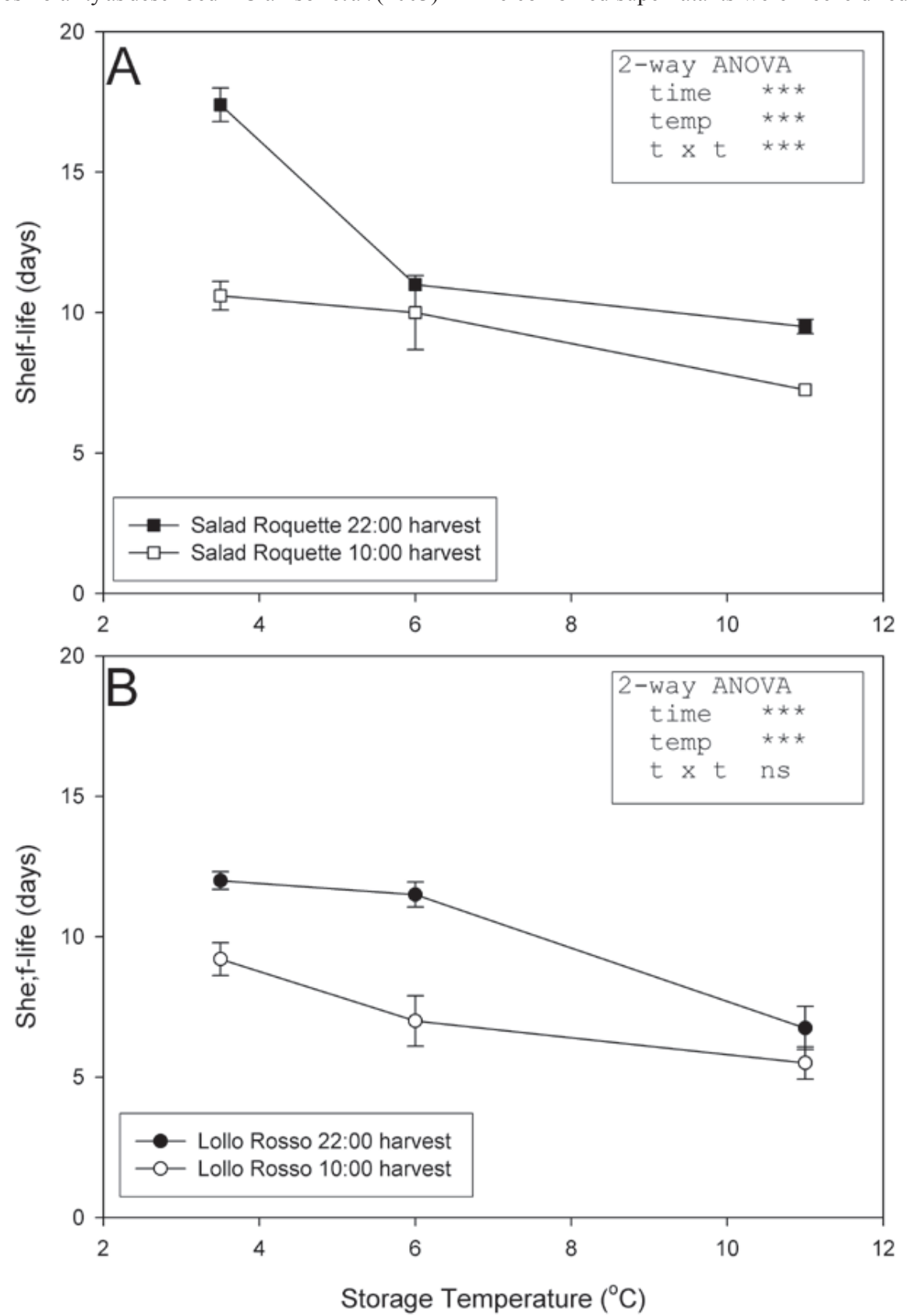

Fig. 1. Shelf life of processed baby leaves of salad roquette (A) and lollo rosso (B) grown at a field site in the U.K. Leaves were harvested at two time points, processed and stored at three temperatures. Shelf life was determined by rejection after two points of breakdown in the bag. Data points represent the mean number of days before rejection (shelf life) \pm SE, $n=5$. to determined the solute potential, $\Psi_{\mathrm{S}}$, with the conversion factor $40 \mathrm{mmol} \cdot \mathrm{kg}^{-1}=0.1 \mathrm{MPa}$ (Van Volkenburgh and Cleland, 1986). Relativeturgor pressure $(P)$ values were determined from the equation, $P=\Psi_{\mathrm{w}}-\Psi_{\mathrm{s}}$.

Experiment 3: Controlled environment trial. Baby leaves of red chard, lollo rosso and salad roquette were sampled at two time points, as the lights came on (start of day) and as the lights went off(end of day) in each room. Leaf disc samples were collected into liquid nitrogen and stored at $-80{ }^{\circ} \mathrm{C}$ for carbohydrate analysis. Soluble carbohydrates were extracted from 2 frozen leaf discs using $80 \%$ ethanol buffered with $50 \mathrm{~mm}$ HEPES-KOH and $5 \mathrm{~mm} \mathrm{MgCl}_{2} \cdot 6 \mathrm{H}_{2} \mathrm{O}$, $\mathrm{pH} 7.4$ for $30 \mathrm{~min}$ at $70{ }^{\circ} \mathrm{C}$. This was repeated five times, pooling the supernatants on ice. The combined supernatants were freeze dried 
for $>24 \mathrm{~h}$, redissolved in sterile distilled water and used to determine the concentration of sucrose using the enzyme-linked assay of Stitt et al. (1989) and a range of known standards. The remaining ethanol insoluble materials were ground to a powder for the analysis of starch levels as described by Stitt et al. (1978) and assayed as for glucose.

Statistical analysis. One and two way analyses of variance (ANOVA) were performed using MINITAB 13.1. Levels of significance, were shown as $F$ values where as $p \leq 0.05$, 0.01 , or 0.001 .

\section{Results}

Experiment 1: U.K. field trial. Baby salad leaves of salad roquette harvested at the end of the day (22:00 HR) had a significantly longer postharvest life than leaves picked the following morning at 10:00 HR (Fig. 1A). At the

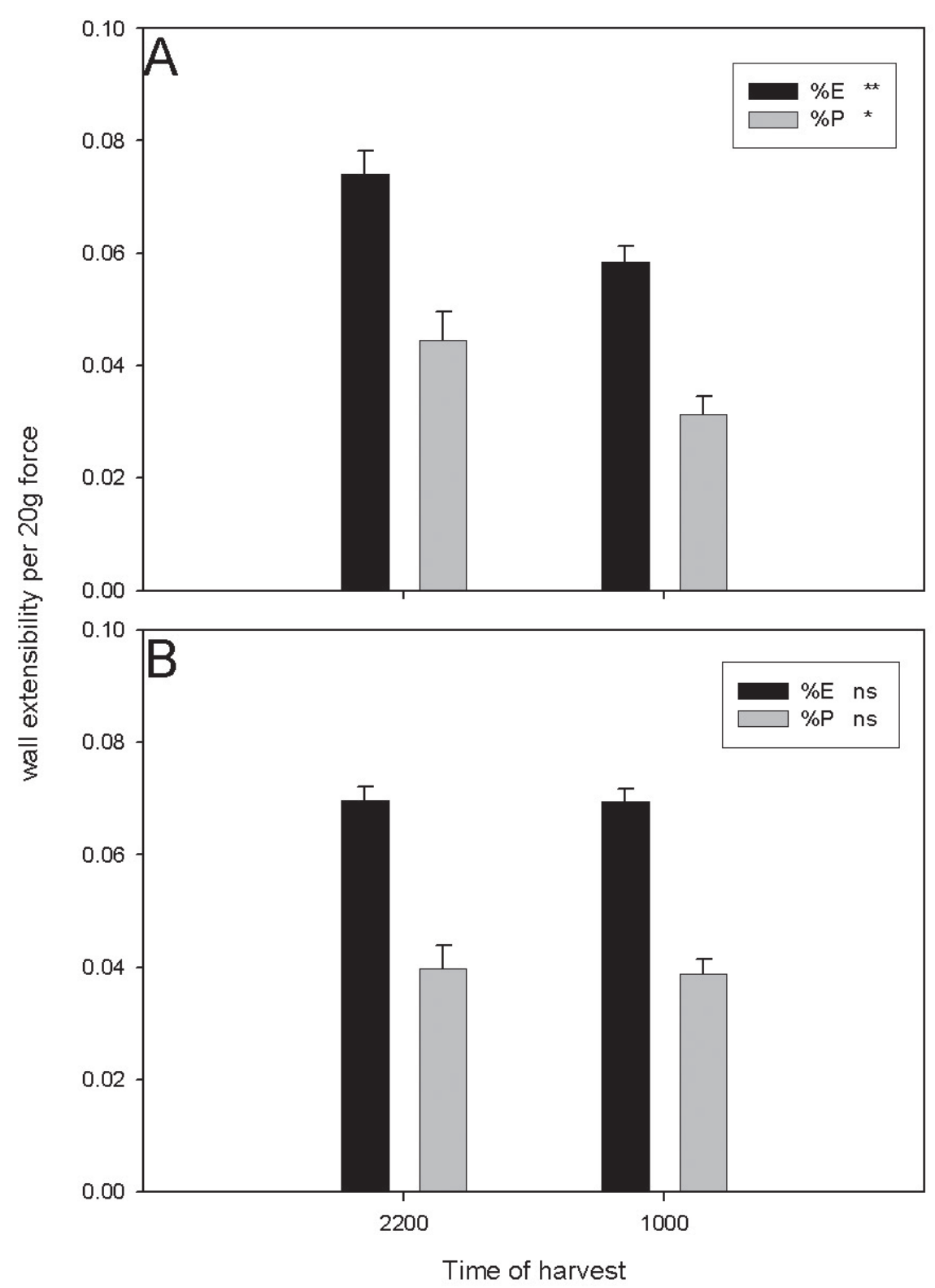

Fig. 2. Cell wall biophysical properties (leaf extensibility) of salad roquette (A) and of lollo rosso (B) grown at a U.K. field site. Leaves were harvested at the time points shown and an Instron apparatus was used to assess percent plasticity $(\% \mathrm{P})$ and percent elasticity $(\% \mathrm{E})$. Data points represent mean extensibility $\pm \mathrm{SE}, \mathrm{n}=25$ or 20 respectively.

lowest storage temperature $\left(3.5^{\circ} \mathrm{C}\right)$ shelf life was extended by $>6 \mathrm{~d}$ from 10.6 to $17.4 \mathrm{~d}$. The shelf life of lollo rosso was also significantly increased by harvest in the evening when compared to the subsequent morning (Fig. 1B). Shelf life is considerably longer in salad roquette than lollo rosso lettuce but the trend of increased postharvest life remained consistent. Longer shelf lives for salad roquette and lollo rosso leaves were shown following storage at lower temperatures. Extended shelf life was seen across the three temperature regimes for all three crops upon evening harvest in comparison to morning harvest the following day. The storage temperature had a significant effect on leaf shelf life.

Extended shelf life following harvest at 22:00 HR was associated with increased leaf cell wall extensibility (Fig. 2), a method used to examine the mechanical properties of the cell wall. Salad roquette harvested at 22:00 HR

HortScience Vol. 40(5) August 2005 had a significantly higher wall extensibility measured as reversible extensibility (percent elasticity) and irreversible extensibility (percent plasticity) when compared to leaves harvested at 10:00 HR that also have a reduced shelf life. Leaves of lollo rosso did not show a difference between these biophysical properties of the leaf dependent on the time of harvest; thus other factors are likely to be associated with extended shelf life following end of day harvest for this species.

Experiment 2: Kenyan field trial. Processability and the effect of the time of harvest findings from Expt. 1 were further investigated in Kenya during January 2002. Shelf life, recorded as days to first sign of breakdown, was measured post processing in commercialscale field grown samples of lollo rosso, salad roquette, and additionally leaves of red chard (Fig. 3). Shelf life was altered significantly by changing the time of harvest in the baby leaves from the morning of processing to the evening before processing. In particular salad roquette shelf life was extended again from 7 $\mathrm{d}$ with harvest at sunrise to $9.1 \mathrm{~d}$ with harvest at sunset the previous evening. Lollo rosso and red chard shelf lives were also extended by using an evening harvest, but the extension was small, averaging $<1 \mathrm{~d}$.

The rates of photosynthesis were examined in situ for field grown leaves of salad roquette and red chard, two species with differing degrees of response to harvest time, to investigate factors that could cause such changes in the shelf life of the baby leaves. Photosynthetic rates and transpiration rates peaked at midday and reached higher levels in salad roquette than those reached by red chard (Table 1), indicating a higher level of photosynthesis in salad roquette associated with a larger response to changes in harvest time.

Baby leaf water relations of red chard and salad roquette were examined at sunset and sunrise to determine leaf water turgor pressure. There was a significant difference between the species for leaf turgor pressure and time of harvest also had a significant effect on leaf turgor pressure. At 18:30 HR salad roquette turgor pressure was $-0.216 \mathrm{MPa} \pm 0.046$ and $-0.055 \mathrm{MPa} \pm 0.009$ at $06: 30 \mathrm{HR}$. For red chard turgor pressure was $-0.347 \mathrm{MPa} \pm 0.058$ at 18:30 $\mathrm{HR}$ and $-0.206 \mathrm{MPa} \pm 0.047$ at 06:30 $\mathrm{HR}$. Turgor pressure in salad roquette leaves was lower compared with red chard, and showed a $75 \%$ reduction in comparison to a $40 \%$ reduction in red chard between evening harvested leaves and morning harvested leaves. These changes in leaf turgor pressure were linked with the extended shelf life shown in evening harvested leaves.

Experiment 3: Controlled environment trial. Controlled growth rooms were used to investigate carbohydrate concentrations following harvest at a start of day and end of day (Fig. 4). Sucrose concentrations were higher at the end of day for salad roquette, lollo rosso and red chard, but significant changes in the concentration of the disaccharide sucrose were apparent between start of day and end of day for salad roquette only (Fig. 4A). The storage carbohydrate starch concentration was not 
significantly changed by the time of harvest (Fig. 4B) but a crop difference remained apparent with concentration of starch significantly higher in lollo rosso at the end of the day. Thus the improvement in shelf life seen at the end of the day in baby salad appeared to be associated with an increase in the concentration of leaf sucrose and not leaf starch.
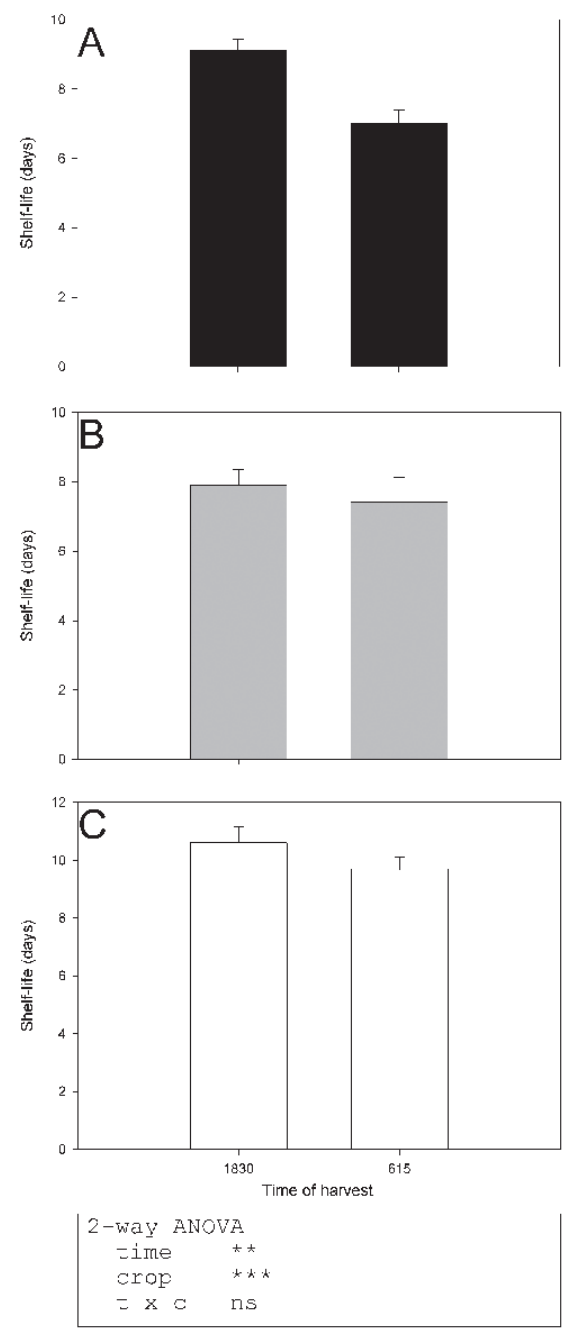

Fig. 3. Shelf life of processed baby leaves salad roquette (A), lollo rosso (B), and red chard $(\mathbf{C})$ grown at a field site in Kenya. Leaves were harvested at two time points (sundown and sunrise), processed and stored at $6{ }^{\circ} \mathrm{C}$. Shelf life was determined by rejection at the first sign of breakdown in the bag following daily examination. Data points represent the mean number of days prior to rejection (shelf life) \pm SE, $n=10$.

Table 1. Peak rates of photosynthesis and transpiration during daylight hours in baby salad leaves of red chard and salad roquette grown at a field site in Kenya. Unshaded leaves orientated to the sun were sampled in situ using a portable photosynthesis system. Data represents the mean net photosynthesis or mean rate of transpiration at the peak sampling point \pm SE, $n=10$.

\begin{tabular}{lcc}
\hline & $\begin{array}{c}\text { Peak } \\
\text { photosynthetic } \\
\text { rate }\end{array}$ & $\begin{array}{c}\text { Peak } \\
\text { transpiration } \\
\text { type }\end{array}$ \\
$\begin{array}{l}\text { rate } \\
\text { Roquette }\end{array}$ & $23.23( \pm 0.779)$ & $4.098( \pm 0.274)$ \\
Red Chard & $17.17( \pm 0.575)$ & $2.512( \pm 0.302)$ \\
\hline
\end{tabular}

\section{Discussion}

The key finding of this research was that postharvest shelf life was correlated to the time of day of harvest in baby leaf salad, and that end of day harvest could increase shelf life by up to $164 \%$ when compared with early morning harvested material. This finding, however, was dependent on species. Extended shelf life was correlated with an accumulation of sucrose during the photoperiod, following photosynthesis, with the largest sucrose accumulation occurring in salad roquette, which also exhibited the largest increase in shelf life compared with lollo rosso and red chard. In contrast lollo rosso leaves accumulated significant amounts of starch during the day while salad roquette starch levels were unchanged, suggesting that the differences among species in sugar metabolism may modulate the potential end of day shelf life extension. Changes in leaf cell wall (for salad roquette) and turgor pressure (salad roquette and red chard) were also observed and contributed to a more processable leaf with extended postharvest shelf life, following end of day harvest. This is an important finding given that most commercial harvest currently occurs during the early morning. There may be considerable potential to improve the shelf life of baby leaf salad crops by rescheduling the time of day of harvest.

The shelf life of lollo rosso, salad roquette and red chard were all increased by an end of day harvest compared to a start of day harvest. The same improvement in shelf life with a harvest later in the day was shown in sweet basil, using visual quality as an indicator of shelf life (Lange and Cameron, 1994), but no underlying mechanisms to explain this difference were elucidated in the study on basil.

There was some evidence that changes in shelf life linked to time of harvest were deter-
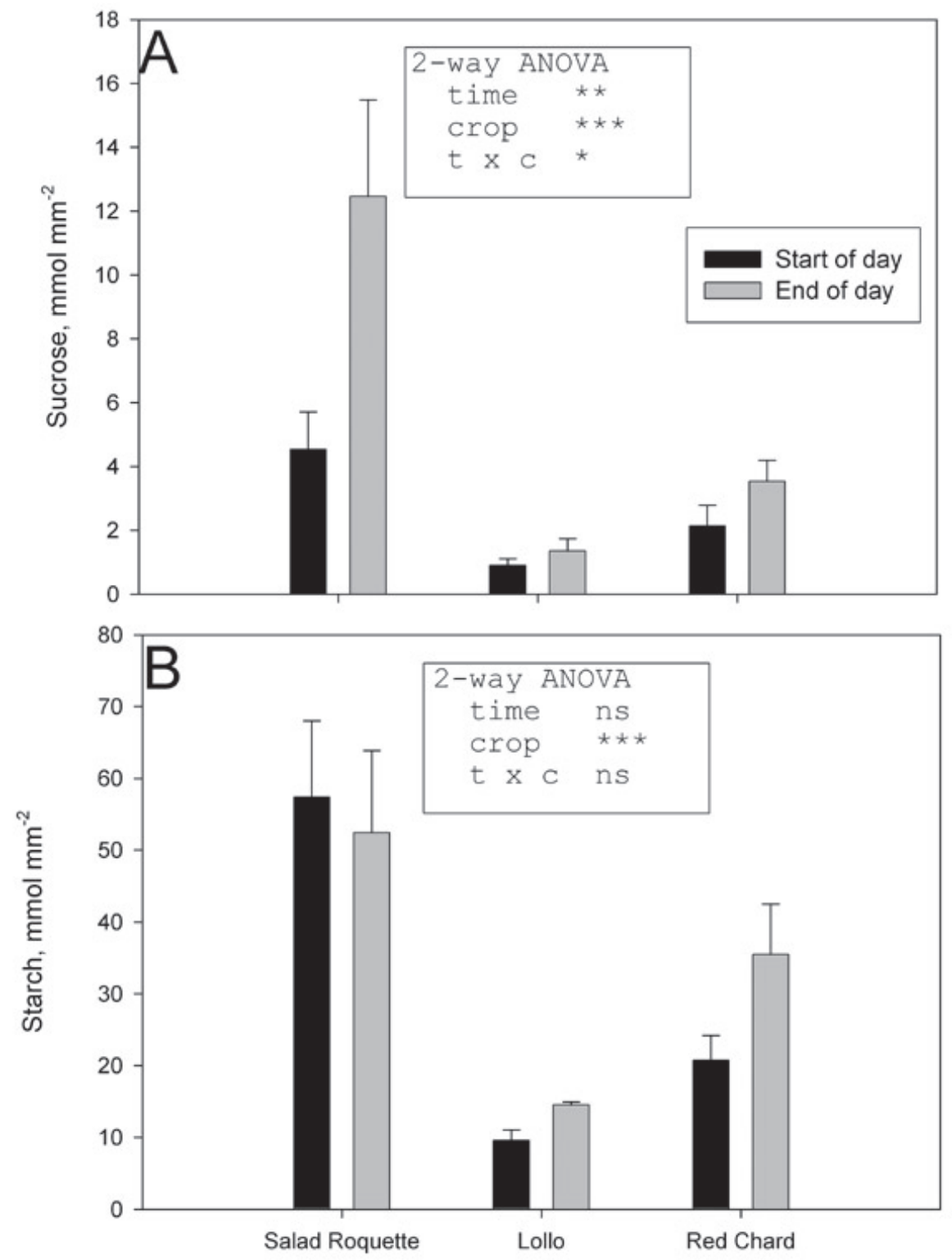

Fig. 4. Sucrose levels (A) and starch levels (B) in baby salad leaves (salad roquette, lollo rosso and red chard) grown in controlled environments. Leaves were harvested as the lights were switched on (start of day) and as the lights were switched off (end of day). Sucrose and starch levels were determined from leaf discs sampled at the same time points using an enzyme linked assay, $\mathrm{n}=6$. 
mined to some extent by altered cell wall biophysical properties, with consistent correlation. It is hypothesized that a leaf with a strengthened cell wall will be more able to withstand the process before reaching break point and degrade slower during storage. For salad roquette in Expt. 1, improved shelf life following end of day harvest (10:00 HR) was correlated with more extensible cell walls confirming our previous findings for traits associated with highly processable leaves (Clarkson et al., 2003). When shelf life was lowest, following morning harvest, cell wall extensibility was also lowest for irreversible extension (percent plasticity), with the more extensible leaves having the longer shelf life, as found with baby salad subjected to salt and mechanical stress (Clarkson et al., 2003). The biophysical properties of the leaves were less sensitive to time of day of harvest in lollo rosso with extensibility not differing between an evening and a morning harvest implying that other traits may be involved in the detected differences in shelf life.

The cell wall modifying protein, xyloglucan endotransglucosylase-hydrolase or XTH(Rose et al., 2002) may be implicated in these patterns of processability and shelf life linked to time of harvest. The XET (xyloglucan endotransglucosylase) activity of XTH may be sensitive to light (Reidy et al., 2001) with night-time activity greater than day-time activity in leaves of Pueraria phaseoloides (Ranasinghe, 1995). We propose that these diurnal changes in cell wall biochemistry may contribute to improvement in baby-leaf processability following evening harvest, at least for some species.

Leaf water relations are also known to follow a characteristic diurnal trend, determined by the opening of stomata in response to light and temperature (Taylor and Davies, 1985). The consequence of this is that turgor pressure may often be higher at the end than the start of day, a factor that may also contribute to the improved processability and shelf life observed for the later harvested leaves. Leaf water relations were cited by King et al. (1982) as a reason for the change in tomato seedling chilling sensitivity at dawn. Perhaps more interesting in the study reported here was the difference in the shelf life observed between red chard and salad roquette, since the former showed a reduced response to time of day for harvest whilst in salad roquette the predicted end of day harvest effect and improved shelf life was apparent. One explanation for this may be linked to the rate of photosynthesis which was consistently higher in leaves of roquette and may have been linked to the accumulation of additional sucrose throughout the day.

Rapid cooling at sunset inhibits the export of sugars from the leaves (Koroleva et al., 2002) and leads to an increased osmotic and turgor pressure in barley leaves and the increased turgor pressure is maintained even with the cooling removed. We have shown that an increased turgor is at least correlated to an improved shelf life and we propose that the rapid cooling in the field during growth in Kenya, as a result of the field location being at the equator with a rapid shift from light to dark and vice-versa, leads to a higher turgor pressure in all the baby salad leaves from Kenya. This then gives rise to an increased postharvest shelf life because more turgid cells are likely to withstand processing and exhibit a long shelf life.

Carbohydrates accumulate during the day as a product of photosynthesis, in particular the disaccharide sucrose with levels of starch remaining constant (Forney and Austin, 1988; Geiger and Servaites, 1994). In lettuce the accumulation of sucrose has a role in $\mathrm{CO}_{2}$ sensitivity, with the leaves most sensitive following an a.m. harvest (Forney and Austin, 1988), and chilling sensitivity is reduced in tomato seedlings with the addition of sugars in the water supply to cut plants (King et al., 1988). Butterhead lettuce types are also more tolerant of storage $\mathrm{CO}_{2}$ when the cultivar has a higher total sugar content(Varoquaux etal. 1996). Our processing and shelflife data support the theory that a higher carbohydrate concentration in the leaf results in an improved postharvest processability and thus shelf life. Here, as with field-grown material in Kenya, no effect of time of day of harvest on either shelf life or leaf sucrose concentration was apparent for red chard, although starch was increased at night in this species. This suggests that there are fundamental differences in the control of carbohydrate metabolism between species and that these will be relevant to the choice of time for harvest.

In summary, this study has shown that time of day of harvest can have a significant impact on the postharvest quality of baby-leaf salad There were some differences in species sensitivity to time of harvest with salad roquette exhibiting most benefit from end-of-day harvest. These data suggest that significant improvements to postharvest shelf life in different environments could be achieved through the rescheduling of the time of day for harvest and also provide relevant information on selection of traits for future genetic improvement.

\section{Literature cited}

Clarkson, G.J.J., E.E. O'Byrne, S.D. Rothwell, and G. Taylor. 2003. Identifying traits to improve postharvest processability in baby leaf salad. Postharvest Biol. Technol. 30:287-298.

Forney, C.F. and R.K. Austin. 1988. Time of day at harvest influences carbohydrate concentration in crisphead lettuce and its sensitivity to high $\mathrm{CO}_{2}$ levels after harvest. J. Amer. Soc. Hort. Sci. 113:581-583

Geiger,D.R. and J.C. Servaites. 1994. Diurnal regulation of photosynthetic carbon metabolism in C-3 plants. Annu. Rev. Plant Physiol. 45:235-256.

King, A.I., M.S. Reid, and B.D. Patterson. 1982. Diurnal changes in the chilling sensitivity of seedlings. Plant Physiol. 70:211-214.

King, A.I., D.C. Joyce, and M.S. Reid. 1988. Role of carbohydrate in diurnal chilling sensitivity of tomato seedlings. Plant Physiol. 86:764-768.

Koroleva, O.A., A.D. Tomos, J. Farrar, and C.J. Pollock. 2002. Changes in osmotic and turgorpressure in response to sugar accumulation in barley source leaves. Planta 215:210-219.

Lange, D.D. and A.C. Cameron. 1994. Postharvest shelf life of sweet basil (Ocimum basilicum). HortScience 29:102-103.

Moccia, S., D. Frezza, and A. Chiesa. 1998. Time of day at harvest effect on postharvest lettuce quality. Agr. Trop. Subtrop. 31:83-86.

Ranasinghe, C.S., 1995. The impact of elevated $\mathrm{CO}_{2}$ and light on leaf growth, cell production and cell expansion, in P. vulgaris and P.phaseoloides. $\mathrm{PhD}$ thesis. Univ. Sussex, Brighton, U.K.

Reidy, B., J. Nösberger, and A. Fleming. 2001. Differential expression of XET-related genes in the leaf elongation zone of F. pratensis. J. Expt. Bot. 52:1847-1856.

Rose, J.K.C., J. Braam, S.C. Fry, and K. Nishitani. 2002. The XTH family of enzymes involved in xyloglucan endotransglucosylation and endohydrolysis: Current perspectives and a new unifying nomenclature. Plant Cell Physiol. 43:1421-1435.

Scholander, P.F., H.T. Hammel, E.D. Bradstreet, and E.A. Hemmingsen. 1965. Sap pressure in vascular plants. Ann. Bot. 79:3-12.

Stitt, M., P.V. Bulpin, and T.A. Rees. 1978. Pathway of starch breakdown in photosynthetic tissues of Pisum sativum. Biochim. Biophys. Acta 544:200-214

Stitt, M., R.M. Lilley, R. Gerhardt, and H.W. Heldt. 1989. Metabolite levels in specific cells and subcellular compartments of plant leaves. Method Enzymol. 174:518-552.

Taylor, G. and W.J. Davies. 1985. The control of leaf growth of Betula and Acer by photoenvironment. New Phytol. 101:259-268.

Van Volkenburgh, E. and R.E. Cleland. 1986. Wall yield threshold and effective turgor in growing bean leaves. Planta 167:37-43.

Varoquaux, P., J Mazollier, and G. Albagnac. 1996. The influence of raw material characteristics on the storage life of fresh-cut butterhead lettuce. Postharvest Biol. Technol. 9:127-139. 